\title{
THE ANALYSIS OF IMPROVED SUSPENSION SYSTEM FOR LOWER LIMB PROSTHESIS
}

\author{
Alina Rodica Spânu ${ }^{1}$, Sorin Cănănău ${ }^{2}$, Daniel Besnea ${ }^{1}$, Nicolae Alexandru Stoica ${ }^{2}$ \\ ${ }_{1}^{1}$ Dept. of Mechatronics and Precision Engineering, University POLITEHNICA of Bucharest, Romania \\ ${ }^{2}$ Dept. of Machine Organs and Tribology Department, University POLITEHNICA of Bucharest, Romania \\ Emails: alina.spanu@upb.ro, sorin.cananau@upb.ro, $\underline{\text { d bes@yahoo.com,nicolae.stoica@upb.ro }}$
}

\begin{abstract}
The suspension system of lower limb prosthesis should be very carefully designed taking into account its main role of accomplishment of firmly contact with the residual limb. The proposed improved solution for the liner was designed as a pellet network inflated with controlled pressure. Consequently, the friction force depends on the pressure values and on the friction coefficient for the material the pellet is made of. The paper concerns about the experimentally measurement of the friction coefficient for the new silicone rubber Zhermack ZA22 Silicone addition RTV2. It should be compulsion on the lower limb prosthesis designer to control the friction force acting on the skin. Otherwise some blisters, pains, circulatory diseases could appear.
\end{abstract}

Keywords: Prosthetics, Silicone Rubber, Molding, Friction Coefficient.

\section{Introduction}

The amputation of lower limb is generally categorized into upper and lower limb, so its range is from the amputation of toes or the ankle to the entire leg. A briefly classification for this amputation type is as above-knee (named trans femoral) and below-knee (called trans tibial) as it is shown in Fig. 1, where we may mention the following parts: 1 - Hip Disarticulation; 2 - Above Knee AmputationTransfemoral; 3 - Below Knee Amputation Transtibial; 4 - Ankle Disarticulation [1].

A person who has suffered the lower limb loss has to experience the only solution of wearing the prosthesis in order to continue the normal life as it was before as well as his physical activities. There are many requirements regarding the design and manufacture of this type of prosthesis because of multiple conditions to attempt such as: the patient comfort during walking, sitting and standing, durability, safety, permanent and adjustable contact with the residual limb.

From the technical design point of view, the lower limb prosthesis has the main parts as they are presented in Fig. 2 [1]: 1 - Socket; 2 - Knee Joint; 3 Pylon. The socket must remain securely coupled to the residual limb and this coupling is referred to as suspension. The suspension system is the most important assembly because it has to provide the firmly contact with the stump. If this condition is not achieved, longitudinal movement and radial clearance may disturb the well-functioning, so that some forces and external mechanical couples could appear especially during walking causing unbalanced walk, which is unsafe, and could affect the other health leg meantime.

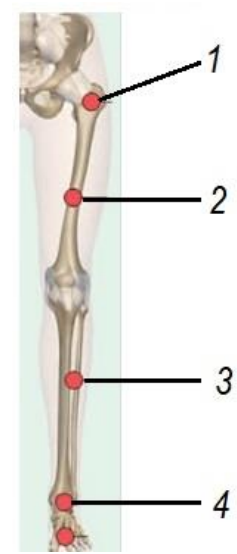

Figure 1: The lower limb prosthesis types [1]

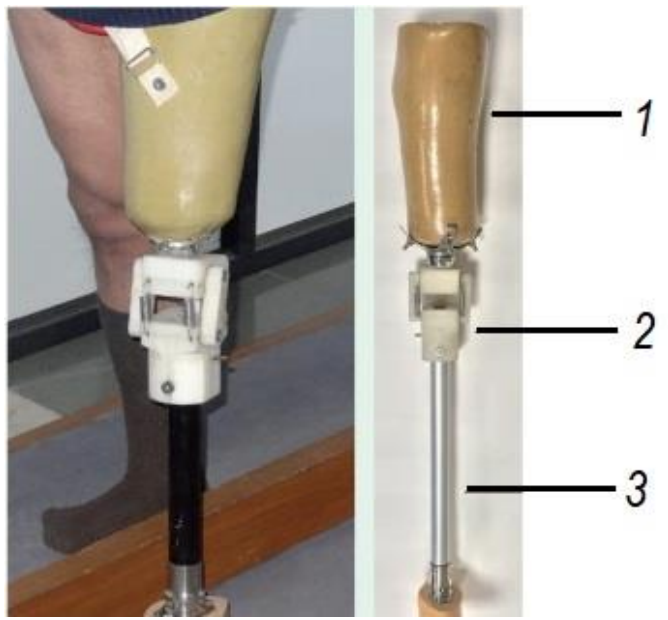

Figure 2: The main parts of lower limb prostheses [1] 
Due to the liner position inside the socket, it plays a decisive role in the interfacial connection between the socket and the residual limb tissue of the patient, so the effective protection of his muscle tissue against excessive pressure and friction should be very well controlled. Otherwise, all sorts of trauma may occur. In the same time, we have to mention here the possibility of volume variation for the residual limb during some daily activities comparing with the volume during the night.

Consequently, there are a lot of different factors that influence the way the contact between the liner and the skin is realized. A solution could be the increasing friction force inside a very well controlled range by using new types of material characterized by special biomechanical properties. There are many studies regarding the material the liner is made of in order to improve their mechanical properties such as static and kinetic friction coefficient, elongation, elasticity and thermal behavior.

The authors of paper [2] described the idea of considering the residuum as a complex material with many components, so the following main tissue types could be considered: skin, adipose, skeletal muscle, tendon and bone. We have to study the nonlinear deformation of soft tissue and high internal strains during prosthetic socket loading. For modeling and computing this nonlinear and viscouselastic behavior, an extension of Hook law with strain-dependent Young's moduli was applied.

The material the liner is made of should be very carefully chosen because of its mechanical parameters that influence the contact based friction, which cause secondary pains and circulatory diseases to the patient. The paper [3] concerned about silver nanoparticles (AgNPs) that showed strong antimicrobial activity and interrupted biofilms formed by bacterial agglomeration as well as their adherence to solid surfaces. AgNPs have been incorporated into polymer composite where they can act as deposits of Ag ions.

When a not properly material of the liner is in direct contact with the skin, the heat generated, the skin transpiration and humidity conditions could favor the bacterial growth especially for immunecompromised people or some other with preexisting disorders. The proposed material, the composite polymer with Ag ions, could be the solution due to its special properties such as psycho-mechanical ones, antimicrobial activity, and cytotoxic effect. Tests were performed to assess hardness, density, compression, and tensile stress for this composite.

Liners are commonly manufactured of silicone elastomers assuring the comfort and soft touch with the tissue as well as good impact absorption. Silicones used in prosthetics are typically polymeric inserts made of silicone as the main raw material with excellent feel smooth.

As we may conclude from the paper [4], some improved methods for the preparation process of the silicone material and for ensuring its mechanics and biosafety could reduce the physical energy consumption, increase wearing time of artificial lower limb and extend the walking distance without any discomfort or supplementary pain.

There are some researches [5] for adding texture to the polypropylene socket that rise the coefficient of static friction, but there were no significant changes for the coefficient of kinetic friction. These textures are printed using 3D printer and the patterns were studied in order to minimize the longitudinal displacement providing a compliant surface suitable for loads transfer during movement.

The paper [6] presents the three types of bionic texture surfaces, which were designed, for two typical prosthetic substrates: silicone rubber and thermoplastic polyurethane elastomer. A study for establishing the optimal texture was made and the conclusions were: for the silicone rubber substrate it was the regular hexagonal prism; for the thermoplastic polyurethane elastomer only the regular quadrangular prism texture satisfied the optimal criteria.

The load transfer implies a pressure force acting permanently. Accordingly to paper [7] the maximum pressure value acting on the skin is about $415 \mathrm{kPa}$ and the resultant shear stresses are $65 \mathrm{kPa}$ for avoiding some circulatory diseases. If the suspension system was based on vacuum, $95 \mathrm{kPa}$ of suction could produce blisters. It has to be taken into account that stresses could be applied in two directions, perpendicular to the surface contact named simply pressure and tangential to it - named stress. The response would be different, so the minimum pressure value $8 \mathrm{kPa}$ is enough for occlude skin blood. Moreover, the muscle tissue is affected sooner than the skin.

Depending on how the shear stress is applied, there are different responses. Blisters and epidermal abrasions appear whether the friction and slip are present. The shear stress without slip between the liner surface and the skin means less injuries and greater volumes are tolerated.

The paper presents the analysis made for an improved suspension system of the lower limb prosthesis in order to experimentally establish the value of the friction coefficient of a new silicone rubber used for the skin contact. The new material named Zhermack is biocompatible and well tolerated for medical equipment.

\section{The Manufacturing of Pellets for the Suspension System.}

The improved suspension system for the lower limb prosthesis consists of a network of pellets distributed around the internal surface of the socket. The main goal is to provide controlled pressure for maintaining the contact with the stump and for preventing from pains and supplementary diseases. The pellets are made of a new material, silicone 
rubber named Zhermack and the producer does not give its friction coefficient yet. The studies showed that it is biocompatible and it has all the properties mentioned before for the silicone suspension.

The pellets have cylindrical shape of $25 \mathrm{~mm}$ diameter and the thickness of $2 \mathrm{~mm}$. They are inflated successively following an established law for a limited period in order to avoid the tissue hurt. Depending on material properties the pressure will accomplish the permanent contact by friction forces and the friction coefficient value will be the most important parameter.

The pellets we have used for the liner were manufactured by the Fused Deposition Modeling (FDM) procedure. During the process, the following stages were accomplished. The preprocessing meaning the design of the CAD model saved as file with .stl extension; the next stage of the work was the sectioning with working planes parallel with the working plane of the 3D printer characterized by its horizontal position. The results were the planar curves, named perimeters which have had the same level. The part is manufactured layer by layer until the entire thickness is achieved. During the last stage, the separation from the platform body is made. The outside working layer, which is the connection between the part and the platform body, is removed. [8,9]

The three-dimensional model for the moulded die is presented in Fig. 3. Because the final part will be empty inside, the solution of using the core with special properties was chosen. As it may be seen in Fig. 4, the core is cylindrical shape and should have enough hardness as it could be placed inside. The procedure with easy fused-model casting was applied with the core made of industrial wax.

The Fig. 5 presents the half moulded die used with the following particular requirements: both the superior and inferior moulded die have enough contact room, so any else object should not be used for attending the dimensional accuracy; a ventilation hole was drilled for air evacuation; it were designed radius and an angle of 5 degrees for assuring the elimination of the part from the casting die. The final moulded core made of industrial wax is presented in Fig. 6.

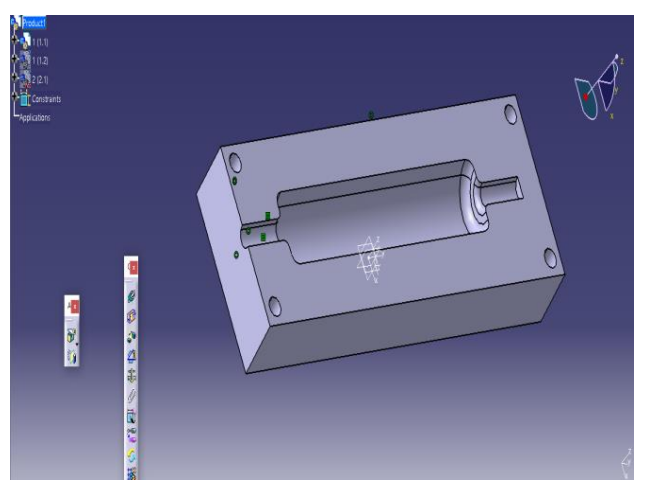

Figure 3: The 3D model for the moulded die

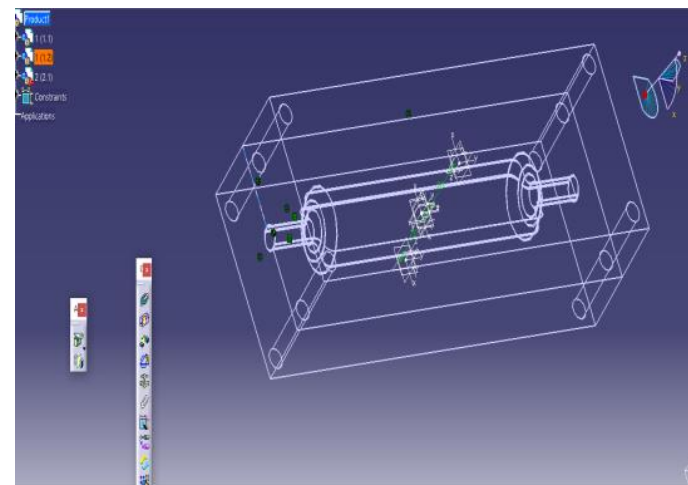

Figure 4: Moulded die for the moulded core made of industrial wax (Wireframe view)

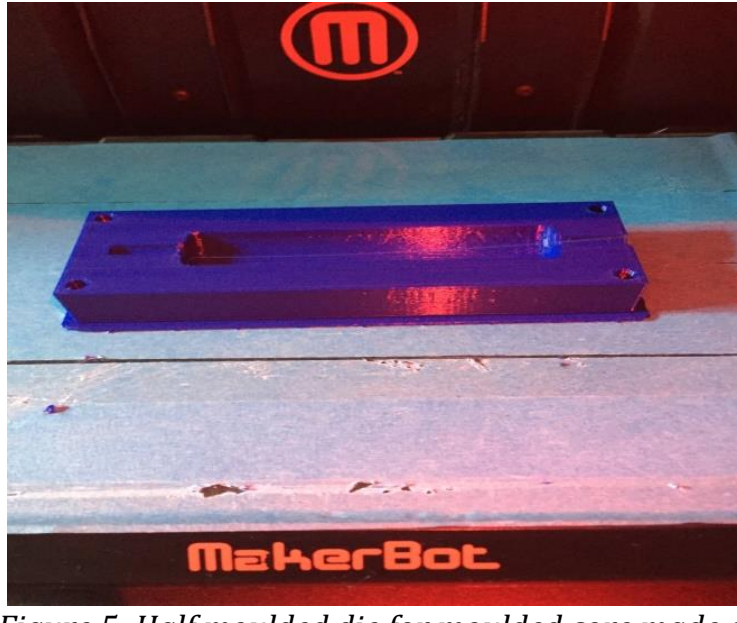

Figure 5: Half moulded die for moulded core made of industrial wax

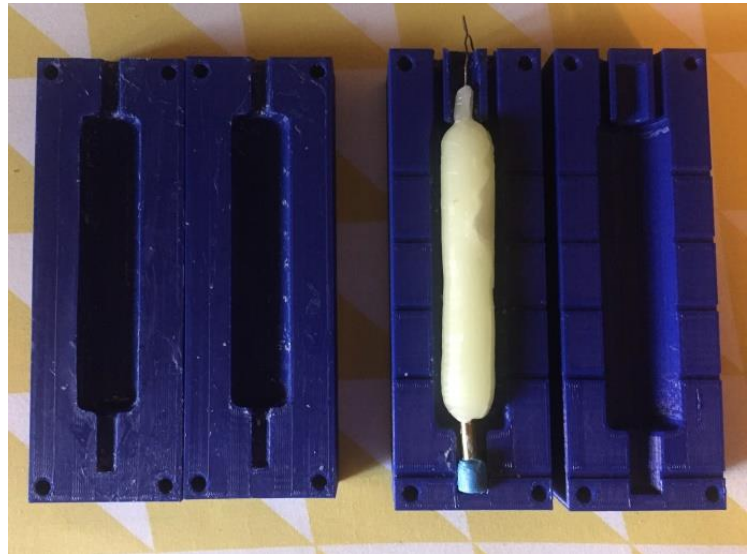

Figure 6: The final moulded core made of industrial wax

The new material, Zhermack ZA22 Silicone adition RTV2 is rubber silicone for addition with two components, which may be used for very complex shapes with a great dimensional stability comprising water and temperature resistance. Its main properties are: great dimensional stability with a variation of $0.01 \%$; for the first 24 hours after the moulding, the dimensional accuracy of the final product is $2 \mu \mathrm{m}$; long term elasticity; working temperature is $1100-3000{ }^{\circ} \mathrm{C}$. Both components of the liquid rubber are mixed together in equal 
percentage and the vulcanization temperature is 200 $-230{ }^{\circ} \mathrm{C}$ lasting 2-3 hours.

The main advantages of this procedure are reffering to the short manufacturing time, the minimum material lost, the complex shapes and low cost.

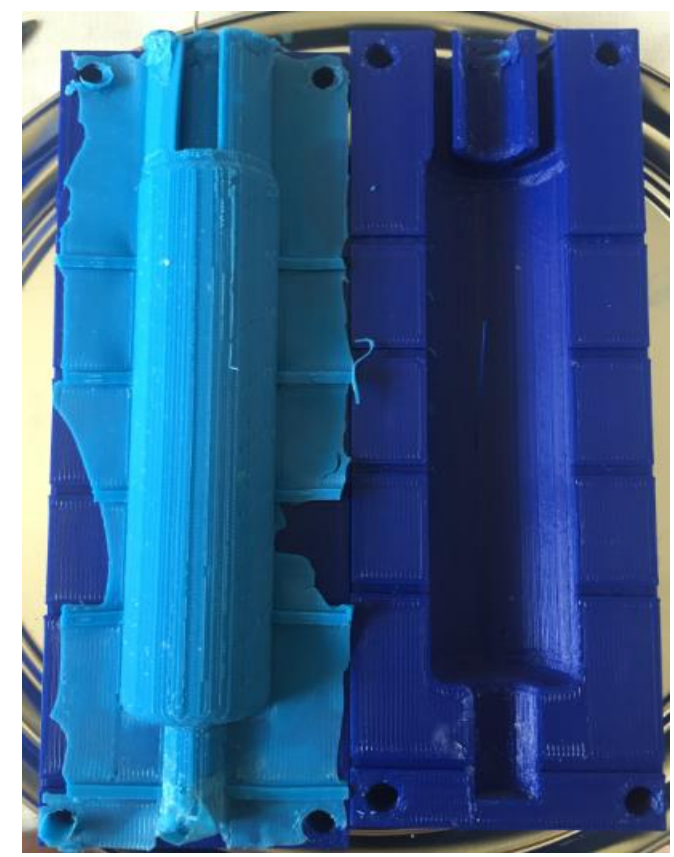

Figure 7: The final pellet with the core

The Fig. 7 presents the final manufactured pellet made of Zhermack ZA22 Silicone addition RTV2 with the core made of industrial wax inside it. The die was manufactured by Rapid prototyping procedure. We have to mention the possibility of leaking the material outside the die because of dimensional errors appeared for both parts as well the working temperature range accordingly to vulcanization temperature. Fig. 8 presents the manufactured pellet with the core removed.

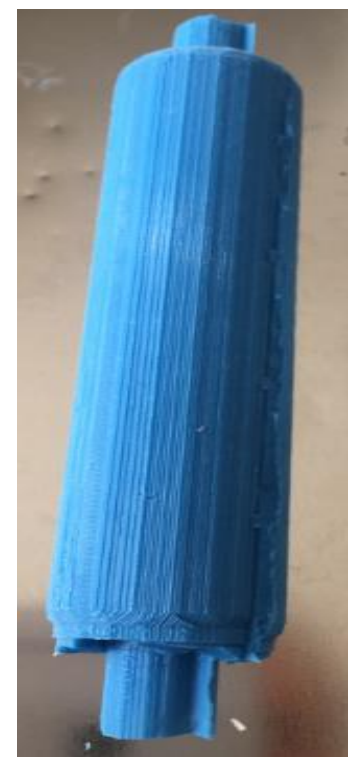

Figure 8: The final pellet with the core removed

\section{Experimental Set-Up}

As it was previously pointed out, the main factor for providing the liner functional parameters must be the friction force developed in the contact area between the pellet and the skin. Accordingly to our proposed solution for the liner, this force depends on the working pressure values inside the pellet network well as on the friction coefficient for the material the pellet is made of.

The Zhermack ZA22 Silicone addition RTV2 material producer did not give any information regarding the friction coefficient value, so we consider the opportunity to experimentally find it out.

The tests presented in the current paper have been conducted on a Bruker (former CETR) UMT-2 Tribometer in order to experimentally establish the evolution of the friction coefficient. The force sensor equipped on the tribometer was a model DFH-5 twodimensional force sensor (range: 0.5 to $50 \mathrm{~N}$, resolution: $2.5 \mathrm{mN}$ ) used to measure the sliding friction force.

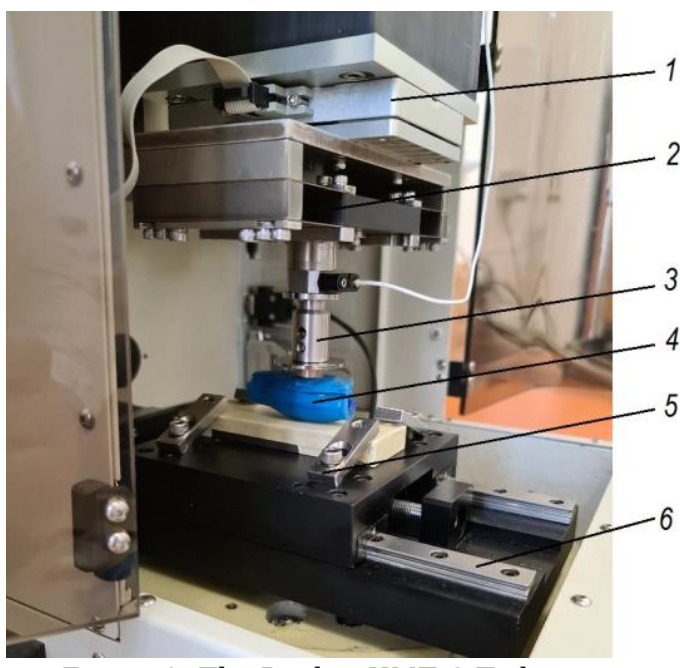

Figure 9: The Bruker UMT-2 Tribometer

Fig. 9 presents the Brucker UMT-2 Tribometer with its main devices we have used for measuring. As we may infer from this figure 1 - Force sensor; 2 - suspension; 3 - upper specimen holder; 4 - the pellet made of Zhermack silicone rubber; 5 - lower specimen holder; 6 - linear motion drive.

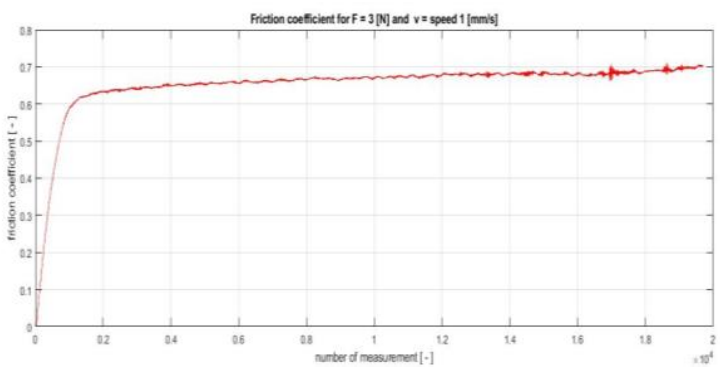

Figure 10: The dependence of friction coefficient versus speed and compressing strain 
The graph chart of the measurement is represented in Fig. 10. The graphic input device for friction coefficient is plotted for the compressing strain $3 \mathrm{~N}$ and linear speed $1 \mathrm{~mm} / \mathrm{s}$.

Table 1.The experimental friction coefficient values

\begin{tabular}{|c|c|c|c|}
\hline $\begin{array}{c}\text { Compression } \\
\text { strain }[\mathrm{N}]\end{array}$ & $\begin{array}{c}\text { Linear } \\
\text { speed } \\
{[\mathrm{mm} / \mathrm{s}]}\end{array}$ & $\begin{array}{c}\text { Friction } \\
\text { coefficient } \\
{[-]}\end{array}$ & $\begin{array}{c}\text { Color } \\
\text { (Fig. 11,12) }\end{array}$ \\
\hline \multirow{3}{*}{3} & 0.1 & 1.15 & \multirow{3}{*}{ red } \\
\hline & 0.5 & 0.98 & \\
\hline & 1 & 0.74 & \\
\hline \multirow{3}{*}{4} & 0.1 & 1.02 & \multirow{3}{*}{ magenta } \\
\hline & 0.5 & 0.9 & \\
\hline & 1 & 0.68 & \\
\hline \multirow{3}{*}{5} & 0.1 & 0.92 & \multirow{3}{*}{ blue } \\
\hline & 0.5 & 0.88 & \\
\hline & 1 & 0.62 & \\
\hline
\end{tabular}

This force was applied on the pellet as it was the subject of translational movement with high accuracy. The measurement comprises three working stages with three values for the linear speed controlled with the linear motion drive of the Tribometer: at first it was $0.1 \mathrm{~mm} / \mathrm{s}$; the second one was $0.5 \mathrm{~mm} / \mathrm{s}$; the third one was $1 \mathrm{~mm} / \mathrm{s}$. Finally, the values of the friction coefficient depending on the compressing strain and linear speed during the translational motion are presented in the Table 1. As we may conclude from Table 1 , the friction coefficient is decreasing directly depending on linear speed for the same compression strain. Analyzing the Fig. 11 and Fig. 12 we may observe the maximum value of the friction coefficient is for the minimum value of speed for the constant compression strain $\mathrm{F}$ $=3 \mathrm{~N}$ and $\mathrm{F} 1=5 \mathrm{~N}$ for instance.

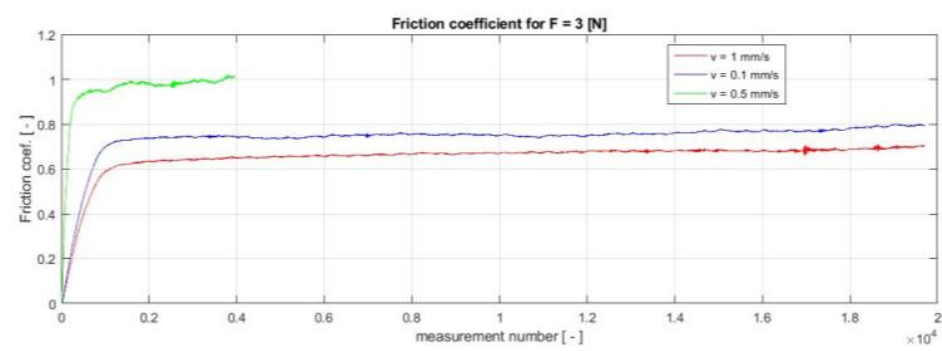

Figure 11: The value for the friction coefficient for the same force and three values of linear speed

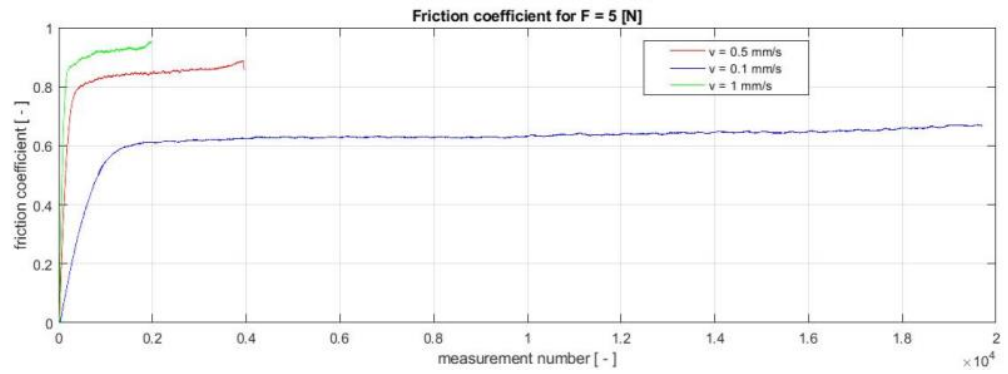

Figure 12: The friction coefficient for $F=5[N]$ and three values of linear speed

The future work will be focused on establishing the relationship between the friction coefficient and strain beyond elastic limit when the above mentioned material is working as strained rubber. Meantime, the working pressure values will be increased and the temperature will be controlled.

\section{Conclusions}

The paper analyzes the mechanical characteristics of the new silicone rubber named Zhermack ZA22 Silicone addition RTV2, the friction coefficient particularly. Taking into account the using of this material for the liner of the lower limb prosthesis, this value should be very carefully recommended because of the direct contact with the skin over a large area, so some diseases should be avoided.

\section{Acknowledgments}

This work was supported by a grant of the Romanian Ministry of Education and Research, CCCDI UEFISCDI, project number 1PTE/2020, cod PN-IIIP2-2.1-PTE-2019-0484, within PNCDI III. The title "Proteză modulară de membru inferior cu sistem de suspensie pneumotronic controlată şi personalizată" („Lower Limb Prosthesis with pneumotronic suspension system controlled and personalized”). 


\section{References}

[1] P. K. Kumar, M. Charan, S. Kanagaraj, "Trends and Challenges in Lower Limb Prostheses", in IEEE Potentials, vol. 36, Issue 1, January - February 2017, Article Number 7814402, pp. 19-23.

[2] David M. Sengeh, Kevin Moerman, Arthur Petron, Hugh Herr, "Multi-material 3-D viscoelastic model of transtibial residuum from in-vivo indentation and MRI Data", in Journal of the Mechanical Behavior of Biomedical Materials, vol. 59, (2016), pp. $379-392$.

[3] Catalina Quintero-Quiroz, Luz E. Botero, Diana Zarate-Trivino, Natalia Acevedo-Yepes, Jorge Saldarriga Escobar, Vera Perez, Luiz Riano, "Synthesis and Characterization of a Silver Nanoparticle-containing Polymer Composite with Antimicrobial Abilities for Application in Prosthetic and Orthotic Devices", in Biomaterials Research, vol. 24, Issue 1, 2020, Article number 13.

[4] Huiru Gu, Huiqin Luan, Zhongjun Mo, Iang Song, Yubo Fan, "Biological and Physical Properties of a Modification Silicone Liner", in IOP Conf. Series: Materials Science and Engineering, vol. 774, (2020) 012110.
[5] Julia Quinlan , Jessica Yohay, Vasanth Subramanian, Brad Poziembo, Stefania Fatone, "Using mechanical testing to assess the effect of lower-limb prosthetic socket texturing on longitudinal suspension", in PLoS ONE, vol. 15, Issue 8 2020, Article Number e0237841

[6] Quanping Feng, Wei Li, Xidong Liu, Wei Ji, Zhongrong Zhou, "Investigation of reciprocating friction characteristics between different bionic surfaces of prosthesis materials and skin", in Biosurface and Biotribology, 2019, vol. 5, Iss. 2, pp. $57-66$.

[7] J. Sanders, Stump-socket interface conditions, in: D. L. Bader, C. V. Bouten, D. COLIN, C. W. Oomens, Pressure Ulcer Research, Springer, 2005.

[8] Petru Berce s.a., Tehnologii de fabricatie prin adaugare de material si aplicatiile lor (Technologies for manufacturing by material adding and their applications), Editura Academiei Romane, Bucuresti, 2014.

[9] Spanu Alina, Victor Constantin, The using of additve manufacturing for prototype productions of moulds, IJOMAM, nr. 1/2017, pp. 7-11.

[10]https://www.zhermack.com/en/product_catego ry/industrial/concrete/rtv-2-silicone-rubber concrete/ 